\title{
Assessment of Post-Laparoscopic Cholecystectomy Pain at Viet Duc Hospital, Vietnam
}

\author{
Dan Thi Nguyen ${ }^{1}$, Huong Thi Thanh Nguyen² \\ ${ }^{1}$ Hanoi Medical University, Hanoi, Vietnam \\ ${ }^{2}$ Faculty of Nursing and Midwifery, Hanoi Medical University, Hanoi, Vietnam \\ Email: nguyendan19@hmu.edu.vn, huongdhy@yahoo.com
}

Received 1 February 2015; accepted 10 March 2015; published 16 March 2015

Copyright (C) 2015 by authors and Scientific Research Publishing Inc.

This work is licensed under the Creative Commons Attribution International License (CC BY). http://creativecommons.org/licenses/by/4.0/

c) (i) Open Access

\begin{abstract}
Gallstones are common and costly in the world. This disease also have high health burden with high mortality. Currently, Laparoscopic Cholecystectomy has become a gold standard for surgical treatment of symptomatic gallstones. Pain is usually the first concern after surgery. Postoperative pain decreases with time but it is severe enough to interfere with daily activities of patients. Pain after the surgery is also affected by many factors. This cross-sectional study aimed to 1) assess pain intensity and interference pain with daily activities; 2) describe the association between pain intensity and interference with daily activities of patients; 3) explore factors that influence postoperative pain after Laparoscopic Cholecystectomy. Data were collected by 50 patients who were undergoing Laparoscopic Cholecystectomy completed questionnaire including the Visual Analogue Scale (VAS) and the Brief Pain Inventory (BPI). Data were analyzed based on three aims. As this is the first time that this type of study is conducted in Vietnam, it is expected that the results will contribute significantly to local knowledge and should add to global perspectives. From understanding the factors that affect severity of pain, this study is expected to design nursing intervention strategies to improve daily activities among patients undergoing LC.
\end{abstract}

\section{Keywords}

Gallstones, Laparoscopic Cholecystectomy, Pain, Intensity, Interference with Daily Activities

\section{Introduction}

Gallstones are common and costly [1]-[4]. About $10 \%$ to $15 \%$ of the adult population in the United States is af- 
fected by this disease [4]. As cited by Ho Thi Diem Thu, the incidence of gallstones was about $5 \%-10 \%$ for Asian countries [5]. It constitutes a major health burden, with cost of gallbladder disease represents a consumption of approximately $\$ 6.2$ billion annually in the US [4]. Gallstones are also a popular disease in Vietnam. The rate gallstones in Vietnam through several community surveys were from $2.14 \%$ to $6.11 \%$ [5].

Laparoscopic Cholecystectomy (LC) has become a gold standard for surgical treatment of symptomatic gallstones [6] [7]. The eminence of LC reflects advantages in surgical morbidity, systemic complications, quality of life, and postoperative pain [5] [8]. In Vietnam, LC was performed widely from central hospitals to provincial hospitals [5]. In Viet Duc hospital, which is one of the largest surgical center of Vietnam, LC is also performed regularly [9] [10].

Although LC has proven to have certain advantages, it is not a pain-free procedure [8]. Some patients after LC still experience considerable discomfort. The pain after laparoscopy is moderate or even severe for some patients, and may require opioid treatment [11]. Postoperative pain decreases with time but it is severe enough to interfere with daily activities in a substantial number of patients [5] [12]-[16]. Pain can result in increased postoperative morbidity and may also prolong the hospital stay-which could increase treatment cost and decrease quality of life of patients [17]. Thus, postoperative pain management remains an important issue [18]. Pain after the surgery is also affected by many factors, such as preoperative pain, anxiety, age, and sex [19]-[22]. Studies have shown that by controlling postoperative pain, comfort of patients undergoing LC could be improved [18] [23]. However, to the limitation of our literature review, the research team could not find any research on pain intensity and interference with daily activities among patients undergoing LC conducted in Vietnam. Therefore, the study entitled "Assessment of post-Laparoscopic Cholecystectomy pain at Viet Duc hospital" has been chosen in order to address three following objectives: 1) to assess pain intensity and interference with daily activities; 2) to describe the association between pain intensity and interference with daily activities; 3) to explore factors that influence postoperative pain after Laparoscopic Cholecystectomy.

\section{Methods}

\subsection{Research Design}

This was a cross-sectional study which used the VAS and BPI to assess pain among patients undergoing LC.

\subsection{Ethics}

Ethics approval was obtained from the Institute of Social And Medical Studies (IRB-ISMS IORG Number 0006663, FWA number 00016762).

The approval to conduct this study was obtained from the Managers of Hanoi Medical University and Viet Duc hospital where the data was collected.

\subsection{Participant Recruitment}

Due to the limitation of time and budget, a convenient sampling method was selected with a sample size of 50 . All patients who were undergoing Laparoscopic Cholecystectomy in data collection time were invited to participate in the study. The inclusion criteria were:

- Patients aged 18 years old and older;

- Patients were diagnosed with gallstones;

- Patients underwent Laparoscopic Cholecystectomy;

- Patients could speak, read and write Vietnamese frequently.

Following that, the exclusion criteria were:

- Patients did not agree to participate in research;

- Patients have participated in this study (patients readmitted during data collection time);

- Patients with stones in the biliary tract;

- Patients acute cholecystitis;

- Patients with other health conditions which need surgical intervention simultaneously.

To recruit participants to the study the researcher contacted the manager of Viet Duc hospital based on the Referral Letter from Hanoi Medical University to describe the study and to obtain Letters of Acceptance. Following that, the researcher had monitored the surgery schedule of all patients in these departments. Therefore, 
the researcher could estimate the timeline to contact patients (at 24 hours postoperative surgery). Participant Information Sheets were sent to all potential participants. Patients who agreed to participate in this study were required to send back this Participant Information Sheet with their signature. Afterward, the researcher spent time for participants to complete the questionnaire. During this time, due to their health status after the surgery, the researcher was sitting beside patients to support them with any reading and writing difficulties if needed.

\subsection{Data Collection Instrument}

In this study, the research team used two tools to assess pain including: Visual Analogue Scale (VAS) and Brief Pain Inventory (BPI). The VAS was used to assess pain before the surgery. The questionnaire have an image of VAS which as a ruler from 0 to 10 . The number 0 indicates no pain; the number 10 indicates worst possible pain. Patients mark their pain score before the surgery in this image.

The BPI was used to assess pain after the surgery. This tool includes two domains: pain intensity (severity) and interference (impact) of pain with daily activities. The first domain is pain intensity to assess pain at its "worst," "least," “average,” and "now”. In this study, a composite (sum) of the four pain items was used to present the postoperative pain intensity of patients. The second domain was used to assess interference of pain with daily activities. This domain measured how much pain has interfered on seven daily activities. According to the BPI guideline, pain interference is typically scored as mean of seven interference items [24]. Three Sub-dimensions of pain interference were proposed: Physical Sub-dimension (walking, general activity, and work), Emotional Sub-dimension (relations with other people, enjoyment of life, and mood), and Sleep. The research team used mean scores of these three Sub-dimensions independently to assess interference with daily activities.

\subsection{Analyses}

Data was analyzed by SPSS version 21. First, descriptive analysis was used to present the characteristics of the participants, intensity and interference of postoperative pain in this study. Independent t-test and analysis of variance (ANOVA) were employed to run bivariate analysis for checking the association between categorical variables and pain intensity variables. Where the significant difference emerged in ANOVA, Post-hoc tests were employed to determine which groups in the sample differ. Pearson correlations were used to examine the associations between overall pain intensity variable and pain interference variables; overall pain intensity variable and factors variables (including age and preoperative LC pain). The $\alpha$-level of 0.05 was selected for all analysis.

\section{Results}

All of 50 patients who were invited to take part in this study fully completed the questionnaire (100\% respondents). Table 1 displays the demographic characteristics of the sample. Females represented $58.0 \%(n=29)$ and the percentage for males was $42 \%(n=21)$. Ratio of female to male was 1.38 . The age range was between 24 and 85 (mean $=54.58, \mathrm{SD}=16.43)$.

About occupation of participants, farmers representing the greatest proportion $(38 \%, n=19)$. Followed by retiree $(24 \%, n=12)$, officers with the percentage of $22 \%(n=11)$. The remaining $16 \%$ was contributed by participants with other occupations. The level of education among participants illustrated that most patients completed high school level $(40.0 \%, n=20)$, followed secondary level with $28 \%(n=14)$. The last two group were patients who have primary level and college/bachelor level, accounted for $14.0 \%(\mathrm{n}=7)$ and $18.0 \%(\mathrm{n}=9)$ respectively.

\subsection{Pain Intensity and Interference with Daily Activities after LC}

\subsubsection{Pain Intensity after LC}

In our study, all patients experienced pain within 24 hours after the surgery (mean $=13.30$, SD = 2.27; possible score ranges from 0 to 40). Figure 1 shown about overall pain intensity after LC.

\subsubsection{Pain Interference with Daily Activities after LC}

Mean scores of these three Sub-dimensions were 18.94 (SD = 2.03), 7.28 (SD = 1.60), and $6.30(\mathrm{SD}=0.65)$ respectively. The overall interference was calculated by sum of the three Sub-dimensions. The mean of overall pain interference of postoperative LC pain with daily activities were 32.52 (SD = 3.38) (Table 2). 
Table 1. Demographic characteristics of samples.

\begin{tabular}{lcc}
\hline Variables & Frequency (N) & Percent (\%) \\
\hline Age in years ( $\mathrm{n}=50)$ & & \\
Range: 24 - 85; mean (SD): 54.58 (16.43) & & \\
Sex ( $=$ 50), Female/male = 1.38 & & \\
Male & 21 & 42.0 \\
Female & 29 & 58.0 \\
Occupation ( $=50)$ & & \\
Farmer & 19 & 38.0 \\
Officers & 11 & 22.0 \\
Retiree & 12 & 24.0 \\
Others & 8 & 16.0 \\
Educational level ( $=50)$ & & \\
Primary & 7 & 14.0 \\
Secondary & 14 & 28.0 \\
High school & 20 & 40.0 \\
College, bachelor & 9 & 18.0 \\
\hline
\end{tabular}

Table 2. Pain interference with daily activities after LC.

\begin{tabular}{lcc}
\hline & Mean & SD \\
\hline Overall pain interference & $\mathbf{3 2 . 5 2}$ & $\mathbf{3 . 3 8}$ \\
Physical Sub-dimension & 18.94 & 2.03 \\
General activity & 4.78 & 0.89 \\
Walking activities & 6.82 & 0.85 \\
Work & 7.34 & 0.72 \\
Emotional Sub-dimension & 7.28 & 1.60 \\
Mood & 4.38 & 0.99 \\
Relations with other people & 1.54 & 0.58 \\
Enjoyment of life & 1.36 & 0.56 \\
Sleep & 6.30 & 0.65 \\
\hline
\end{tabular}

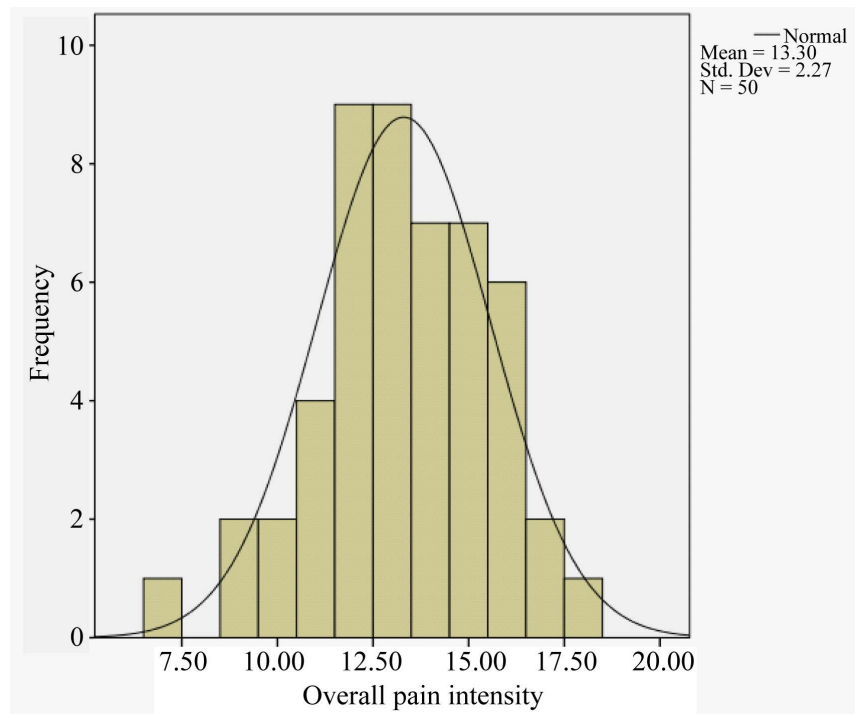

Figure 1. Overall postoperative pain intensity after LC. 


\subsection{The Association between Pain Intensity and Interference with Daily Activities after LC}

Our study found that patients who experienced postoperative pain, their physical activities would be limited and their sleep could also be affected. However, postoperative pain did not influence emotion of patients (Table 3).

\subsection{Factors Affecting Pain after LC}

Bivariate analysis was used to examine the relationship between factors including age, sex, occupation, educational level, preoperative pain, fear of surgical procedure and overall postoperative pain intensity. Our results found that preoperative pain and fear of surgical procedure were significant predictors of postoperative pain. Females were more likely experience postoperative pain than males. Nonetheless, there were no significant differences between overall postoperative pain intensity with age, occupation, and educational level. Table 4 summaries the bivariate relationships between these factors and overall pain intensity.

Table 3. The association between pain intensity and interference with daily activities after LC.

\begin{tabular}{ccc}
\hline & Mean (SD) & Pearson correlation \\
\hline Overall pain interference & $32.52(3.38)$ & $0.54^{* * *}$ \\
Physical sub-dimension & $18.94(2.03)$ & $0.61^{* * *}$ \\
Emotional sub-dimension & $7.28(1.60)$ & 0.21 \\
Sleep & $6.30(0.65)$ & $0.38^{* *}$ \\
\hline
\end{tabular}

${ }^{* *} \mathrm{p}<0.01 ; \stackrel{* * *}{\mathrm{p}}<0.001$.

Table 4. Bivariate analysis of factors that influence postoperative LC pain.

\begin{tabular}{|c|c|c|}
\hline Variables & Mean of factors & Bivariate analysis \\
\hline Age & $54.58(16.43)$ & $0.07_{(P)}$ \\
\hline Sex & & $-5.45^{* * *}(T)$ \\
\hline Male & $11.67(1.85)$ & \\
\hline Female & $14.48(1.77)$ & \\
\hline Occupation & & $0.72_{(A)}$ \\
\hline Farmers & $13.47(2.20)$ & \\
\hline Officers & $13.09(2.51)$ & \\
\hline Retiree & $13.83(1.90)$ & \\
\hline Others & $12.38(2.27)$ & \\
\hline Educational level & & $1.44_{(A)}$ \\
\hline Primary & $12.71(2.36)$ & \\
\hline Secondary & $13.86(2.38)$ & \\
\hline High school & $12.70(2.32)$ & \\
\hline College, bachelor & $14.22(1.64)$ & \\
\hline Preoperative pain & $2.92(1.21)$ & $0.30_{(P)}^{*}$ \\
\hline Fear of surgical procedure & & $18.29^{* * *}{ }_{(A)}$ \\
\hline None & $10.50(2.52)$ & \\
\hline Little & $11.89(1.60)$ & \\
\hline Average & $13.71(1.44)$ & \\
\hline Multiple & $15.50(1.40)$ & \\
\hline
\end{tabular}

${ }^{*} \mathrm{p}<0.05 ;{ }^{* * *} \mathrm{p}<0.001 ;{ }_{(\mathrm{T})} \mathrm{T}$-test; (A) One Way Anova; (P) Pearson correlation. 


\section{Discussion}

\subsection{Pain Intensity and Interference with Daily Activities after LC}

\subsubsection{Pain Intensity after LC}

In our study, all patients experienced pain within 24 hours after the surgery, even though it was not severe pain (mean $=13.30$; possible score ranges from 0 to 40 ). This result could be explained that all patients in this study was used at least one type of analgesics after the surgery. Previous studies showed a similar fact that pain is the most concerned problem after operation which always requires at least a pain management method [11] [22] [25]. Dabbagh indicated that patients may experience significant abdominal disturbances and pain particularly during the first 24 hours postoperatively. Their study also reported that more than $30 \%$ of patients experienced a longer treatment time due to postoperative pain [26]. Another study was conducted on LC postoperative pain among 382 patients. The results indicated that pain was the most frequent symptom, both before and after the operation [27]. Joris et al. indicated that LC is not a free-pain procedure where most of patients even experience moderate or even severe pain [11]. The higher level of pain found their study compare to our study may due to the different pain relief method was applied or may due to different pain measure scale was used in data collection.

\subsubsection{Pain Interference with Daily Activities after LC}

Although postoperative pain decreased with time, it was severe enough to interfere with daily activities in a substantial number of patients [12]. Our study has shown that overall postoperative pain had interfered with daily activities (mean $=32.52$; possible score ranges from 0 to 70 ). In which, physical activities and sleep were interfered more than emotional activities. These results were consistent with previous studies where indicated that the presence of pain was associated with trends towards poorer psychological functioning and social integration. The intensity of pain was associated with interference with a number of important basic daily activities [14].

In addition, Girard et al. reported that the most interference of pain with daily activities were on sleep, normal work, and walking ability in percentages ranging from 40 to 55\% of patients reported moderate-to-severe interference. General activity and mood were also affected. Relationship with other people, enjoyment of life, appetite, and concentration were the least affected (23 to 32\% of patients reported moderate to severe interference) [15]. In Vietnam, Van Vu Van et al. also indicated that Physical activity and functions were more interference than emotions and perceptions [16].

\subsection{The Association between Pain Intensity and Interference with Daily Activities after LC}

Our results showed that there was a positive correlation between pain intensity and pain interference with daily activities. A number of studies have investigated the impacts of postoperative pain on daily life activities, and results are similar. In which, levels of physical activities were usually limited after operation due to pain [13]. The intensity of pain was associated with interference on a number of important basic daily activities such as sleep, mood, mobility, and self-care activities [14]. Van Vu Van et al. indicated that the interference of pain worse to the quality of life in all aspects of the survey with average score quality of life was 53.7/100 [16].

Literature also revealed that the presence of pain was associated with trends towards poorer psychological functioning and social integration [14]. However the research team could not find the association between postoperative pain intensity and emotional activities. This may due to the different conceptions of quality of life in the Western countries compared with developing countries. In developing countries, the majority populations are laborers. The low socio-economic situation has resulted in lower perceptions of quality of life especially mood, relations with other people and enjoyment of life. This finding is consistent with a study conducted in Vietnam where the results also indicated that physical activity and functions were more interference than emotions and perceptions [16].

\subsection{Factors Affecting Pain after LC}

In this study, the research team found that preoperative pain, fear of surgical procedure and sex were significant predictors of postoperative pain. This is consistent with the factors influencing pain in other studies.

In our study, patients with higher level of preoperative pain intensity would experience higher level of post- 
operative pain. Similarly, Omari et al. indicated that postoperative pain in patients who have preoperative pain was more severe than those without preoperative pain [19].

Our study also found that there were a positive association between fear of surgical procedure and postoperative LC pain intensity. This finding is consistent with literature which showed that fear of surgical procedure may increase postoperative LC pain intensity. Shirin et al. illustrated that the patients in the intervention group who received preoperative anxiety intervention before surgery showed lower pain scores than the patients who did not receive preoperative anxiety intervention [28].

Our results indicated that female patients experienced more postoperative pain than male patients. This finding is identical to previous studies which showed that pain was significantly higher in female than male patients [26] [27]. It is because female patients are usually more sensitive to pain than male patients as explained by Van Vu et al. [16]. Pain tolerance of women is often less than men [21].

In this study, there were no significant differences between postoperative pain intensity with age, occupation, and educational level. Nonetheless, literature revealed that age was identified as independent risk factors for early LC postoperative pain [22]. Omari et al. also revealed that younger patients reported postoperative sensitivity twice as frequently as older patients [19]. This difference may be due to a small sample size and most of patients in our study were middle age.

\subsection{Limitation of This Study}

There present study also contains some limitations that may affect the generalizability of the results. Selection bias may have occurred since a convenience sample was used. The sample size of this study was not calculated based on a statistical formula; the number of participants was set based on the limitation of time and the budget of a bachelor graduation thesis. In addition, this study used cross-sectional design without a follow up; this study only assessed the intensity and interference of pain in LC patient within 24 hours after the surgery, while the intensity and interference of postoperative pain could change over time.

\section{Conclusion}

Although most patients experience minor postoperative pain, patients still experience daily activities interference. In which, pain intensity affect physical activities and sleep but not affect emotions of patients. Preoperative pain and fear of surgical procedure were significant predictors of postoperative pain. Females were more likely to experience postoperative pain than males.

\section{Implication}

The findings of this study have potential to meaningfully contribute to nursing care plan for patients undergoing LC in Vietnam. As postoperative pain significantly affects physical activities and sleep, nursing interventions should focus on supporting patients' physical daily activities as well as increasing quality of patients' sleep after surgery. Female patients usually have more postoperative pain than male patients. Therefore, nursing interventions should pay more attentions on female patients after surgery. Patients with higher level of preoperative pain severity would experience higher level of postoperative pain. Thus, nursing interventions should consider more intensive care for patients who have more preoperative pain.

Our study also indicated that more fear of surgical procedure may increase postoperative pain intensity. Therefore, it is recommended that nurses should provide more health education to patients before the surgery such as giving patients with more information about the surgery, listen to the patients and motivate them. Consequently, patients could feel more comfortable while in the hospital.

Finally, the Brief Pain Inventory tool is recommended to assess intensity and interference of postoperative pain for nurses who taking care patients undergoing surgery. However, nurses must consider modifying emotional items in the BPI to make them more suitable with conception of quality of life among Vietnamese patients.

\section{Acknowledgements}

The authors would like to thank the Training and Manage Boards of Hanoi Medical University have created conditions in many ways for our study. We would like to sincerely thank Dr. Charles S. Cleeland, Professor of 
Cancer Research at the University of Texas in the United States, who gave us the permission to use the BPI tool. We would like to express our heartfelt thanks go to all patients after Laparoscopic Cholecystectomy at Viet Duc hospital in answering questionnaires. Without those answers, this study would not be possible.

\section{References}

[1] Shaffer, E.A. (2006) Epidemiology of Gallbladder Stone Disease. Best Practice \& Research Clinical Gastroenterology, 20, 981-996. http://dx.doi.org/10.1016/j.bpg.2006.05.004

[2] Tazuma, S. (2006) Gallstone Disease: Epidemiology, Pathogenesis, and Classification of Biliary Stones (Common Bile Duct and Intrahepatic). Best Practice \& Research Clinical Gastroenterology, 20, 1075-1083. http://dx.doi.org/10.1016/j.bpg.2006.05.009

[3] Pitchumoni, C.S. (2010) Increasing Prevalence of Gallstones; Diagnostic and Therapeutic Options. Medicine Update, 20, 486-490.

[4] Stinton, L.M. and Shaffer, E.A. (2012) Epidemiology of Gallbladder Disease: Cholelithiasis and Cancer. Gut and Liver, 6, 172-187.

[5] Thu, H.T.D. (2014) Quality of Life in Patients after Laparoscopic Cholecystectomy for Gallstone Disease. Ho Chi Minh Medicine and Pharmacy University: Hepatobiliary Surgery.

[6] Barut, I., et al. (2005) Experience of Ambulatory Laparoscopic Cholecystectomy in Turkish Patients. European Journal of General Medicine, 2, 96-99.

[7] Tsimoyiannis, E.C., et al. (1998) Intraperitoneal Normal Saline Infusion for Postoperative Pain after Laparoscopic Cholecystectomy. World Journal of Surgery, 22, 824-828. http://dx.doi.org/10.1007/s002689900477

[8] Wills, V.L. and Hunt, D.R. (2000) Pain after Laparoscopic Cholecystectomy. British Journal of Surgery, 87, $273-284$. http://dx.doi.org/10.1046/j.1365-2168.2000.01374.x

[9] Hepatobiliary Surgery Department (2014) In-Out Patient Management Records. Viet Duc Hospital.

[10] Emergency Gastrointestinal Surgery Department (2014) In-Out Patient Management Records. Viet Duc Hospital.

[11] Joris, J., Thiry, E., Paris, P., Weerts, J. and Lamy, M. (1995) Pain after Laparoscopic Cholecystectomy: Characteristics and Effect of Intraperitoneal Bupivacaine. Anesthesia \& Analgesia, 81, 379-384.

[12] Beauregard, L., Pomp, A. and Choinière, M. (2004) Severity and Impact of Pain after Day-Surgery. Canadian Journal of Anaesthesia, 45, 304-311. http://dx.doi.org/10.1007/BF03012019

[13] Ochroch, E.A., Gottschalk, A., Troxel, A.B. and Farrar, J.T. (2006) Women Suffer More Short and Long-Term Pain than Men after Major Thoracotomy. Clinical Journal of Pain, 22, 491-498. http://dx.doi.org/10.1097/01.ajp.0000208246.18251.f2

[14] Jensen, M.P., Hoffman, A.J. and Cardenas, D.D. (2005) Chronic Pain in Individuals with Spinal Cord Injury: A Survey and Longitudinal Study. Spinal Cord, 43, 704-712. http://dx.doi.org/10.1038/sj.sc.3101777

[15] Girard, F., Chouinard, P., Boudreault, D., Poirier, C., Richard, C., Ruel, M. and Ferraro, P. (2006) Prevalence and Impact of Pain on the Quality of Life of Lung Transplant Recipients: A Prospective Observational Study. Chest, 130, 1535-1540. http://dx.doi.org/10.1378/chest.130.5.1535

[16] Vu, V.V., Hanh, V.T.X., Giang, P.T.T. and Hoang, T.T.H. (2010) Studying the Situation of Pain and Quality of Life in Advanced Cancer Patients at HCMC Oncology Hospital. The Medicine Journal of Ho Chi Minh City, 14, 811-822.

[17] Robinson, T.N., Biffl, W.L., Moore, E.E., Heimbach, J.K., Calkins, C.M. and Burch, J.M. (2002) Predicting Failure of Outpatient Laparoscopic Cholecystectomy. American Journal of Surgery, 184, 515-518. http://dx.doi.org/10.1016/S0002-9610(02)01080-2

[18] Bisgaard, T., Klarskov, B., Rosenberg, J. and Kehlet, H. (2001) Factors Determining Convalescence after Uncomplicated Laparoscopic Cholecystectomy. JAMA Surgery, 136, 917-921. http://dx.doi.org/10.1001/archsurg.136.8.917

[19] Al-Omari, Q.D., Al-Omari, W.M. and Omar, R. (2009) Factors Associated with Postoperative Sensitivity of Amalgam Restorations. Journal of the Irish Dental Association, 55, 87-91.

[20] Aubrun, F., Valade, N., Coriat, P. and Riou, B. (2008) Predictive Factors of Severe Postoperative Pain in the Postanesthesia Care Unit. Anesthesia \& Analgesia, 106, 1535-1541. http://dx.doi.org/10.1213/ane.0b013e318168b2ce

[21] de Silva, L.M., Kakuda, C.M., de Campos Vieira Abib, A., Fugiwara, F.Y., Lima e Lara, G.F., Mazzotta, R.C., et al. (2013) Factors Associated to Postoperative Pain in the Post-Anesthetic Care Unit in Patients Submitted to Laparoscopic Gastroplasty. Revista Dor, São Paulo, 14, 239-244.

[22] Bisgaard, T., Klarskov, B., Rosenberg, J. and Kehlet, H. (2001) Characteristics and Prediction of Early Pain after Laparoscopic Cholecystectomy. Pain, 90, 261-269. http://dx.doi.org/10.1016/S0304-3959(00)00406-1

[23] Gupta, A., Thörn, S.E., Axelsson, K., Larsson, L.G., Agren, G., Holmström, B. and Rawal, N. (2002) Postoperative 
Pain Relief Using Intermittent Injections of 0.5\% Ropivacaine through a Catheter after Laparoscopic Cholecystectomy. Anesthesia \& Analgesia, 95, 450-456.

[24] Charles, S. and Cleeland, P. (2009) The Brief Pain Inventory User Guide. The University of Texas MD Anderson Cancer Center. http://www.mdanderson.org/

[25] Vezakis, A., Davides, D., Gibson, J.S., Moore, M.R., Shah, H., Larvin, M. and McMahon, M.J. (1999) Randomized Comparison between Low Pressure Laparoscopic Cholecystectomy and Gasless Laparoscopic Cholecystectomy. Surgical Endoscopy, 13, 890-893. http://dx.doi.org/10.1007/s004649901127

[26] Dabbagh, A.A. (2009) Pain after Laparoscopic Cholecystectomy. Zanco Journal of Medical Sciences, 13, 37-42.

[27] Ure, B.M., Troidl, H., Spangenberger, W., Dietrich, A., Lefering, R. and Neugebauer, E. (1994) Pain after Laparoscopic Cholecystectomy. Surgical Endoscopy, 8, 90-96. http://dx.doi.org/10.1007/BF00316616

[28] Bakr, S.A., Ali, S.K. and Khudhr, S.A. (2010) Impact of Preoperative Anxiety Intervention on Postoperative Pain. Zanco Journal of Medical Sciences, 14, 72-77. 